\title{
Does Technology Matter? How Digital Self-Efficacy Affects the Relationship between ICT Exposure and Job Dissatisfaction
}

\author{
Julian Busse \\ University of Goettingen \\ julian.busse@uni-goettingen.de
}

\author{
Robin Busse \\ University of Goettingen \\ robin.busse@uni-goettingen.de
}

\author{
Matthias Schumann \\ University of Goettingen \\ mschuma1@uni-goettingen.de
}

\begin{abstract}
Despite multiple potentials of information and communication technologies (ICT), their increasing diffusion at today's workplaces may lead to psychological issues for employees, unveiling a dark side of ICT use. Our study aims to examine the association between work-related ICT exposure (i.e. ICT use and digital work intensification) and job dissatisfaction. We further look at the role of digital self-efficacy as a moderator of the effect of digital work intensification. Cross-sectional data from a nationally representative study of 1,145 employees were used in multiple regression analysis. Our results show that higher levels of digital work intensification are associated with higher levels of job dissatisfaction. Further, digital self-efficacy buffers the effect of digital work intensification on job dissatisfaction. Thus, our findings imply that fostering employees' confidence in their abilities in dealing with the challenges of digitalization promotes employees' job satisfaction and coping with the negative effects of work-related ICT exposure.
\end{abstract}

\section{Introduction}

The use of information and communication technologies (ICT) pervades the work and life in the twenty-first century and influences the way we communicate, interact, learn and work [1-3]. Indisputably, organizations have gained great advantages in productivity, efficiency, and flexibility through the implementation and assimilation of ICT [4, 5]. However, these benefits are also countered by a supposedly "dark side" of the extensive use of ICT [1, 6-8]. From the perspective of the employees, exposure to ICT requires significant cognitive, social, and physical skills [1], potentially leading employees to experience stress when using ICT [2, 6]. Since the organizational use of ICT has become ubiquitous, real-time, and functionally pervasive, users are often challenged to process information simultaneously and continually from multiple applications [9]. Therefore, prior research reveals that users of ICT have to deal with a surfeit of information, experience frequent interruptions, and engage in multitasking on different computing devices [9]. Consequently, the intensive use of ICT increases work time and work speed leading to an overall work intensification that may have negative effects on individuals' cognitive, psychological and physical health $[3,5]$. For example, previous studies have shown associations between ICT use and job satisfaction [2, 10], burnout and exhaustion [3], job performance [9], and organizational commitment [2].

To describe the "negative" implications of ICT use at work, the term "technostress" has gained increasing popularity in various disciplines, such as information systems (IS) and psychology [11]. First coined in 1982, the term describes an ineffective coping with technology that results in distress [12]. However, higher job demands are not necessarily bad for employees' well-being, especially when the job demands are balanced with adequate resources $[2,11]$ like social support or ICT-related support [2, 13]. Furthermore, individual resources like self-efficacy or technology commitment are discussed to mitigate the negative effect of ICT-related stress [4, 14]. Therefore, instead of technostress, we use the term ICT exposure as a more general and neutral term to cover what previous studies have called technostress, digital stress, or ICT demands.

A theory that has been widely used to study the conditions under which ICT exposure is experienced negatively is Lazarus' transaction theory of stress from organizational psychology [5]. Within the model of technostress, the transaction theory emphasizes that stress can result from a combination of a demand condition that causes the stress (stress creators) and the individual's psychological response to the stress creators (manifest adverse outcomes referred to as 'strain') [1, 11, 15]. Even if an increasing number of studies have shed light on this set of relationships from different angles in recent years [5], there are still open questions that need further exploration for a better understanding of the conditions under which ICT use represents a concern for employees' well-being. In this paper, we focus on three open research gaps in particular. 
(1) The use of ICT extremely varies in the working population among occupational characteristics [11]. However, only a few studies draw on occupational characteristics that influence ICT-related work processes [e.g., 16]. Most studies were collected in several occupations and therefore, neither allow separate views on specific occupations nor provide information on the effects of specific ICT tasks [3]. An additional limiting factor is that some of the samples were made up of employees from only one or a small number of companies [e.g., 2, 7]. Representative samples are rarely used [17] so that in most cases generalized conclusions for occupational groups are not possible running the risk of biased analysis.

(2) With regard to the lack of studies on occupational differences, it has already been indicated that there is also a lack of sufficient concretization of ICT use in this context. Depending on the respective occupation, users work with different kinds of ICT systems [3]. However, previous studies mostly measure the level of ICT use or technology use in general [e.g., $1,11,18]$. The consequences of this one-sided view are that only limited implications for science and practice can be derived from a general level of ICT use. Thus, it is not possible to assess whether specific systems or groups of systems are the sources for a high ICT exposure in a specific occupational group.

(3) Furthermore, very limited research has been done so far in the organizational behavior and IS on how to reduce ICT-related exposure [14]. However, understanding the sources of negative psychological and behavioral reactions toward the use of ICT is very important [19] to be able to initiate interventions that reduce the ICT exposure of ICT users. In response to this demand, IS executives and researchers are particularly interested in the effect of digital self-efficacy, a relatively new construct in research that encompasses the perception of one's digital abilities as well as the conviction of coping with digital requirements, to explain and reduce the negative effects of ICT exposure on employees' well-being [14]. However, the research on the association of digital self-efficacy and ICT exposure with regard to strains like job dissatisfaction is still very limited.

To address these research gaps, the focus of our study is on work-related ICT exposure to understand its implications for employees' job dissatisfaction. In examining work-related ICT exposure, we distinguish between digital work intensification on the one hand and the use of occupation-specific ICT on the other hand. Digital work intensification refers to the individual perception of employees that the use of ICT leads to a higher workload, requires multitasking, or to work faster [16]. However, digital work intensification may depend on the extent to which employees work with ICT, as the effects of ICT are a function of the degree to which ICT are used at the workplace. It is expected that frequent ICT users are more exposed to the effects of ICT than occasional users [1]. Consequently, it is necessary to control for the level of occupation-specific ICT use. Therefore, we are also interested in the relationship between the use of occupation-specific ICT and employees' job dissatisfaction. This distinction leads us to our first research question:

RQ1: In what way is ICT exposure (distinguishing the two aspects occupation-specific ICT use and digital work intensification) associated with employees' job dissatisfaction?

Research suggested that ICT-mediated working tasks are particularly stressful for those employees that lack the [technical] wherewithal [8]. This assumption implies that if the appropriate resources are in place, the consequences of ICT exposure can be mitigated. As mentioned before, different mechanisms like the digital self-efficacy of employees can alleviate the effects of ICT exposure [14]. Since there is evidence that people with higher self-efficacy are more likely to feel able to cope with difficult or challenging situations and are therefore more likely to face these situations [20], a buffering effect of the concept of self-efficacy can be postulated. Therefore, to the extent that ICT exposure influences employees' job dissatisfaction, it is likely to have a greater impact on employees with a low digital self-efficacy.

Since digital self-efficacy is a personal disposition, the focus of interest is particularly on its relationship to employees' individual perceptions of stress caused by digital work intensification. However, research hardly takes into account how digital self-efficacy impacts job dissatisfaction in the context of digital work intensification. Thus, our second research question is:

RQ2: To what extent does digital self-efficacy influence the impact of digital work intensification on job dissatisfaction?

Responding to these two research questions, we strive for a quantitative study with a national representative sample of commercial and service occupations, which account for about $30 \%$ of all employees in the German labor market. To assess the data, we use multivariate regression techniques.

The paper is organized as follows. After this introduction, we describe the theoretical background of our research and derive our hypotheses. Then, we present our research model and research methods. Afterward, we outline and discuss our study results. Finally, we conclude by highlighting the research and practical contributions of our research and its limitations. 


\section{Theorizing the association between ICT exposure, digital self-efficacy and job dissatisfaction}

\subsection{Job dissatisfaction}

Job satisfaction is an important strain construct in the research on the dark side of ICT use $[5,21]$ and a major determinant for employees' well-being [22]. Locke defines job satisfaction "as an outcome of the perception that one's job fulfills the important values that are congruent with one's needs" [23]. There is practical evidence that job dissatisfaction is one of various negative end-user reactions to ICT [2, 12]. For example, the use of ICT can lead to employees feeling overloaded or insecure about how to use the systems, which can lead to stress and consequently increase job dissatisfaction [4]. Furthermore, job dissatisfaction can lead to absenteeism and turnover and, therefore, affects other organizational outcomes [2]. Consequently, any changes in job dissatisfaction as a result of ICT exposure are an important outcome to measure. For these reasons, we consider job dissatisfaction as our central, dependent construct.

\subsection{ICT exposure: digital work intensification and occupation-specific ICT use}

A central goal of current research on the use of ICT in the working context is focused on exploring whether or not the use of ICT contributes to a more fragmented work experience and, if so, what the consequences of this more fragmented work experience might be for enterprises and their employees [16]. In particular, researchers identified two effects that are related to the use of ICT in the working context: work extension [16] and work intensification [24]. Work extension refers to the extent employees need to work even in non-work time and space in ways that imply that work never stops, and creates a sense of presenteeism [25].

In the current study, we focus on digital work intensification, which appears when the use of ICT leads to a more intense work experience that is often characterized by the effect that one must consistently work faster, work more, or perform different tasks simultaneously [16]. In contrast to the process of work extension, work intensification can be experienced even if total work hours are unchanged [16]. From a historical point of view, the intensification of work has been an influential trend that was already indicated by trend data during the 1990s in Europe [26] and the United States [27]. In the 21 st century, the increasing diffusion of ICT systems in organizations continues to shape this effect as several studies indicate a positive relationship between ICT use and work intensification [e.g., 24, 26, 28]. Nowadays, employees in commercial and service occupations need to handle a wide range of enterprise application systems, of which customer relationship management systems or enterprise resource planning systems are just two very prominent examples [8, 29, 30]. In addition, most of the employees use office applications (e.g., word processing or spreadsheet editors), which are part of the standard software equipment of every office workplace. Furthermore, digital communication in the form of video conferencing, in particular, has become more relevant due to the remote work during the COVID-19 pandemic [31] and supplements the portfolio of digital forms of communication alongside e-mail and messenger services.

However, the effects of (digital) work intensification are by no means clear-cut, as both positive and negative effects have been observed [11]. For example, studies have demonstrated increased productivity as a positive outcome of a general work intensification [32]. On the other side of the coin, digital work intensification has also been associated with several negative outcomes for employees. Employees' stress levels, as well as occupational health, are two concepts that have been the subject of considerable research $[16,17,24]$. Yet, the extent to which the intensification of digital work has an impact on job dissatisfaction has been hardly studied to date. Research on work intensification that did not explicitly consider the effect of ICT use found that job dissatisfaction increased over time when work intensification increased as well [24, 32]. Furthermore, research on technostress observed a positive association between technostress creators and job dissatisfaction with the magnitude varying from small to medium among these studies [2, 21]. A limitation of these studies is, however, that they only took into account the overall effect of technostress creators on outcomes like job dissatisfaction [21]. Hence, there is an epistemic interest in the association between ICT exposure (distinguishing the two aspects digital work intensification and occupation-specific ICT use) and job dissatisfaction $[11,21]$. This argumentation leads us to draw the following hypothesis:

Hypothesis 1a: Higher levels of digital work intensification relate to higher individuals' job dissatisfaction.

To control the effect of digital work intensification for the level of occupation-specific ICT use, we also examine the relationship between ICT use and job dissatisfaction and postulating the same association. Thus:

Hypothesis 1b: Higher levels of occupation-specific ICT use relate to higher individuals' job dissatisfaction. 


\subsection{Digital self-efficacy}

The understanding of the construct of self-efficacy has its roots in the social cognitive theory originated by Bandura [33]. Self-efficacy is conceptualized as the self-perception of one's capabilities to organize and execute courses of action required to attain designated types of performance, which is concerned not with the skills one has but with judgments of what one can do with whatever skills one possesses [34]. Research has demonstrated that individuals with higher self-efficacy take on effort to a greater extent to cope with those situations that require new behaviors or place high demands on the individuals, whereas those with lower self-efficacy tend to engage in fewer challenging activities [20, 34].

A large body of research has focused on the association between self-efficacy and performance in various academic domains and found a strong, positive relationship [35]. For example, research has found that self-efficacy mitigates the negative effects of work stress on work performance in the organizational context [36]. In this respect, it is not surprising that the concept of self-efficacy has already been the subject of several studies in connection with the use of ICT. In some cases, the concept was specified to certain facets like self-efficacy in dealing with computers $[35,37]$ or the internet [20]. In other cases, the concept was used more globally, for example, the self-efficacy in dealing with new technologies $[8,36]$ or as a general ICT self-efficacy [4]. To summarize, there is evidence that individuals with greater confidence in their ability to use ICT, technologies, computers, or the internet will experience less stress. For this study, we aggregate these aspects as digital self-efficacy, defined as the self-perception of one's abilities to cope with the requirements of digital work [35, 38].

However, to the best of our knowledge, there has been little research on whether digital self-efficacy can buffer the negative impact of ICT related stress at the workplace. Therefore, we examine the moderating effects of digital self-efficacy on the relationship between digital work intensification and employees' job dissatisfaction.

Based on the previous research arguments, we assume that employees with higher digital self-efficacy are more confident in coping with the challenges of digital work. Consequently, higher self-efficacy can lower the negative effects resulting from increasing digital work intensification caused by the use of ICT. Hence,

Hypothesis 2: Digital self-efficacy buffers the effect of digital work intensification on job dissatisfaction.

\section{Research model and methods}

The research model is developed on the assumption that ICT exposure is positively related to employees' job dissatisfaction.

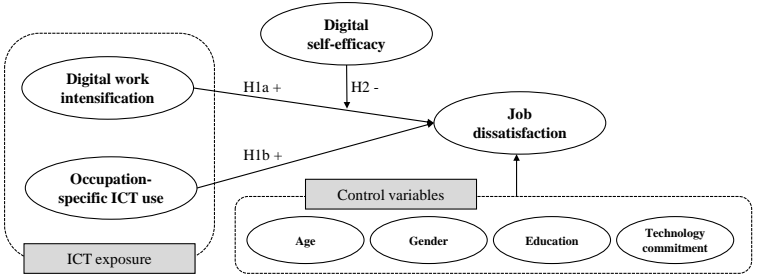

Figure 1. Research model of associations between ICT exposure, digital self-efficacy and job dissatisfaction

In addition, it is argued that digital self-efficacy moderates the relationship between digital work intensification and employees' job dissatisfaction. Furthermore, the research model controls for age, gender, education, and technology commitment. Figure 1 illustrates the research model.

\subsection{Data collection and participants}

To reach a national representative sample of commercial and service occupations, we used a quantitative approach. We collected the data through a structured online survey in February 2020 via an ISO-certified (ISO 26362) online panel (www.respondi.com). This panel recruits its participants via offline and online methods. It ensures high quality through minimizing participation frequency, focusing on intrinsic motivation instead of financial dependency, and conducting continuous controls. In the sample, we included women and men between 18 and 60 years that worked full-time or part-time. To ensure the representativity of the sample with the German labor market, we aimed to reach a quote of $30 \%$ to $40 \%$ of commercial and service occupations in the whole sample. To increase data quality, we implemented four "knock-out" criteria [39]. After data cleaning, the sample consisted of 3,020 people, of which 1,145 work in commercial and service occupations (37.91\%).

Figure 2 relates the sample obtained to the population according to the employment statistics of the Federal Employment Agency of Germany [40]. People between the age of 25 to 55, people in full-time occupations as well as people with an academic degree are somewhat overrepresented in our sample. Nevertheless, there is a good fit between our sample and the population above all characteristics, which allows us to draw valid statements about the population of German service and commercial occupations. 


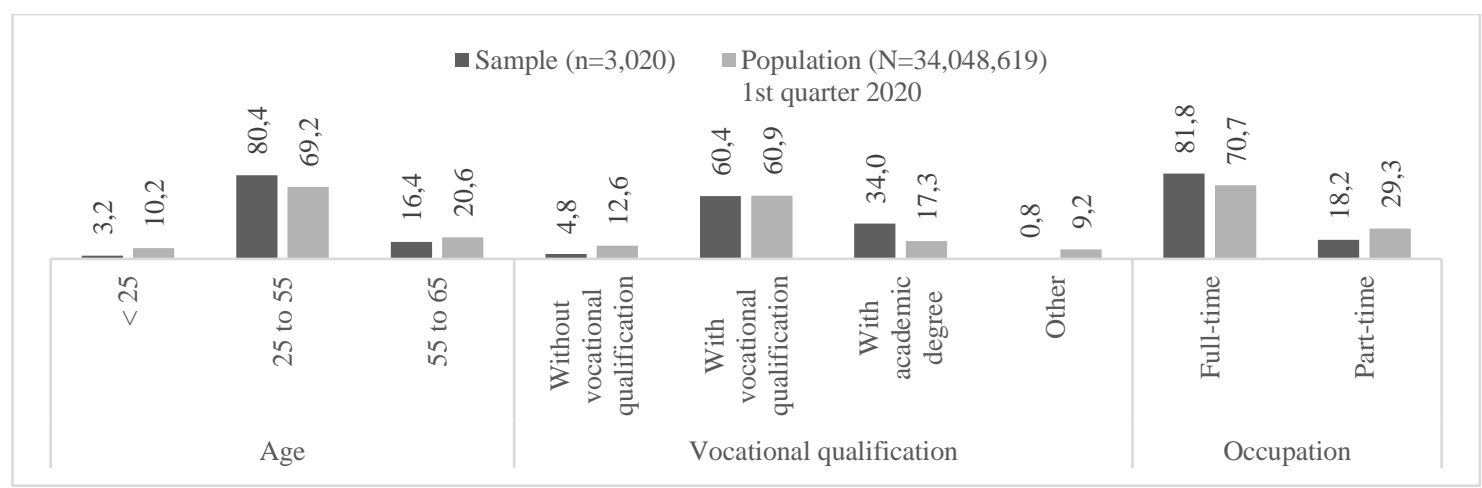

Figure 2. Representativity of obtained sample

Regarding the relevant sample group of commercial and service occupations $(\mathrm{n}=1,145)$, participants were approximately 42 years old, $61 \%$ were female and $30 \%$ had at least a bachelor's degree and above. In terms of job experience in service and commercial occupations, $54 \%$ had an experience of greater than 10 years. This result suggests that incumbents have had enough time to experience all the work characteristics present in the job.

\subsection{Measurement and scale development}

To ensure content validity, we adapted the constructs from literature. Items were measured by a 4-point Likert scale ranging from 1 ("strongly disagree") to 4 ("strongly agree"). Moreover, we calculated Cronbach's alpha (CA) per construct to evaluate their internal consistency [41].

We measured employees' job dissatisfaction, the dependent variable of the study, using four items of the job dissatisfaction scale of Enzmann \& Kleiber [42] (a representative item is: "I do not enjoy my job"). The average $(\mathrm{M}=1.71, \mathrm{SD}=0.67)$ of the four items produced a reliable scale $(\alpha=0.81)$. As a further sensitivity analysis, the model fit of the scale was assessed using Mplus [43], and likewise found to be $\operatorname{good}\left(\chi^{2}(2)=9.950, \mathrm{p}=0.007, \mathrm{RMSEA}=0.059\right.$, $\mathrm{CFI}=0.999$, TLI $=0.996)$.

Concerning the independent variables of the study, digital work intensification was measured with six items adapted from Borle et al. [11] and the German version of the Work Design Questionnaire [44]. Three items each refer to the use of information systems and the use of communication systems as sources of digital work intensification (e.g., "Due to the use of information systems (communication systems), I have to perform different tasks simultaneously"). The average ( $\mathrm{M}=$ $2.29, \mathrm{SD}=0.79)$ of the six items produced a reliable scale $(\alpha=0.91)$.

To measure the use of occupation-specific ICT, we differ between six different categories of ICT at typical workplaces of commercial and service occupations: (1) office applications $(\mathrm{M}=2.81, \quad \mathrm{SD}=1.05), \quad$ (2) enterprise applications for finance $(\mathrm{M}=1.51$, $\mathrm{SD}=0.76)$, (3) enterprise applications for production $(\mathrm{M}=1.27, \mathrm{SD}=0.56)$, (4) enterprise applications for sales $(\mathrm{M}=1.71, \mathrm{SD}=0.89),(5)$ enterprise applications for resource planning $(\mathrm{M}=1.66, \mathrm{SD}=0.84)$, and (6) communication applications $(\mathrm{M}=2.28, \quad \mathrm{SD}=0.61)$. The categories were measured by questions as to the frequency of work-related use of occupation-specific ICT. For instance, the first category office applications was measured by asking three questions about the extent to which employees use word processing, spreadsheet, and presentation software. The categories then were computed as a mean score with 1 indicating low and 4 indicating high use.

\section{Table 1. Distribution of dependent and independent variables}

\begin{tabular}{lcc}
\hline Variables & $\mathrm{M}$ & $\mathrm{SD}$ \\
\hline $\begin{array}{l}\text { Dependent variables } \\
\text { Job dissatisfaction }\end{array}$ & 1.71 & 0.67 \\
\hline Independent variables & & \\
Digital work intensification & 2.29 & 0.79 \\
Office applications & 2.81 & 1.05 \\
Communication applications & 2.28 & 0.61 \\
Enterprise applications for & & \\
$\quad$ finance & 1.51 & 0.76 \\
$\quad$ production & 1.27 & 0.56 \\
$\quad$ sales & 1.71 & 0.89 \\
$\quad$ resource planning & 1.66 & 0.84 \\
Digital self-efficacy & 3.45 & 0.64 \\
\hline
\end{tabular}


The moderating variable in our study digital self-efficacy was measured with four items (e.g., "I fear that the changes brought by digitalization will overwhelm me.") adapted from the general self-efficacy scale of Jerusalem \& Schwarzer [45]. After inverting the four items, the average $(\mathrm{M}=3.45, \mathrm{SD}=0.64)$ of the items produced a reliable scale $(\alpha=0.87)$. High values on the scale indicate high digital self-efficacy.

In addition to the main explanatory factors, we control the vocational degree. In vertical order, we differentiate between no vocational degree $(3.06 \%$ of our sample), vocational education and training (VET) degree (67.07\%), and university degree (29.87\%). Also, we control for respondents' highest school degree (no or lower secondary degree $4.0 \%$ of our sample, intermediate-secondary degree $36.3 \%$, upper-secondary degree $59.7 \%$ ). Moreover, we account for technology commitment with nine items (e.g. "I quickly take a liking to new technical developments") from the corresponding scale of Neyer et al. [46]. Other control variables include gender and age.

\subsection{Analytical strategy}

To examine how far employees' job dissatisfaction is shaped by ICT exposure and digital self-efficacy, we apply hierarchical linear regression models. To determine how far the effects of digital work intensification are influenced by occupation-specific ICT use, technology commitment, and other confounding variables, we estimate different hierarchical models (Hypothesis 1a \& 1b). To shed light on a buffering effect of digital self-efficacy, we introduce an interaction between the measures for digital work intensification and digital self-efficacy to the regression model. This allows us to investigate buffering and accelerating effects of digital self-efficacy on the relationship between employees' perceived digital work intensification and job dissatisfaction (Hypothesis 2).

\section{Results}

In the following section, we describe the central findings from our analyses regarding the hypotheses proposed in this paper, beginning with the major findings of correlation analyses. As expected, higher levels of digital work intensification correlate with higher values of each of the considered forms of occupation-specific ICT (Pearson $r<0.3$, p $<0.01$ ). Furthermore, digital-self efficacy positively correlates with the control variable technology commitment $(r=.48, \mathrm{p}<0.01)$, and negatively with digital work intensification $(r=-0.18, \mathrm{p}<0.01)$. The latter indicates that employees with a higher digital self-efficacy perceive lower levels of digital work intensification.

The following multivariate analyses aim to examine to what extent employees' job dissatisfaction varies as a function of ICT exposure and digital self-efficacy when other important control variables are controlled.

The intention of Model 1 is to determine to what extent employees' job dissatisfaction is shaped by perceived ICT exposure when no other variables are controlled.

Table 2. Determinants of employees' job dissatisfaction

\begin{tabular}{|c|c|c|c|c|c|c|}
\hline & \multicolumn{2}{|l|}{ Model 1} & \multicolumn{2}{|c|}{$\begin{array}{l}\text { Model } 2 \\
(+ \text { controls })\end{array}$} & \multicolumn{2}{|c|}{$\begin{array}{l}\text { Model } 3 \\
\text { (+digital self-efficacy) }\end{array}$} \\
\hline & $\mathrm{B}$ & SD & $\mathrm{B}$ & SD & $\mathrm{B}$ & SD \\
\hline \multicolumn{7}{|l|}{ ICT exposure } \\
\hline Digital work intensification & $0.188 * * *$ & 0.026 & $0.180 * * *$ & 0.026 & $0.099 * * *$ & 0.025 \\
\hline Office applications & $-0.043^{*}$ & 0.020 & -0.032 & 0.020 & -0.017 & 0.018 \\
\hline Communication applications & $-0.112 * *$ & 0.036 & -0.066 & 0.036 & $-0.075^{*}$ & 0.033 \\
\hline \multicolumn{7}{|l|}{ Enterprise applications for } \\
\hline finance & -0.037 & 0.030 & -0.027 & 0.029 & -0.039 & 0.027 \\
\hline production & $0.130 * *$ & 0.047 & $0.115 * *$ & 0.046 & 0.046 & 0.043 \\
\hline sales & -0.049 & 0.026 & -0.034 & 0.026 & -0.016 & 0.024 \\
\hline resource planning & -0.006 & 0.030 & -0.009 & 0.029 & 0.007 & 0.027 \\
\hline \multicolumn{7}{|l|}{ Moderator } \\
\hline Digital self-efficacy & & & & & $-0.455 * * *$ & 0.033 \\
\hline Controls & & & $\checkmark$ & & $\checkmark$ & \\
\hline $\mathrm{N}$ & 1,145 & & 1,145 & & 1,145 & \\
\hline Adjusted R ${ }^{2}$ & 0.053 & & 0.106 & & 0.234 & \\
\hline
\end{tabular}

Note. Unstandardized coefficients. Level of significance: ${ }^{*} p<0.05 ;{ }^{* *} p<0.01$; ${ }^{* \star *} p<0.001$. Model 2 and 3 control for age, gender, education (vet and high school degree), and technology commitment. The variance inflation factors (VIFs) in our various model tests were less than 2 - well below the threshold of 10, indicating that multicollinearity is not a concern. 
Approximately $5.3 \%$ of the variance is explained by digital work intensification and the use of occupationspecific ICT. Model 1 shows that increased intensity of digital work is associated with higher job dissatisfaction, even when the intensity of occupationspecific ICT use is controlled.

In contrast to Model 1, Model 2 further controls for employees' age, gender, education, and technology commitment. Adding these confounders to the model significantly improved the model fit (indicated by the likelihood ratio test $[p<0.001])$. In Model 2, approximately $10.6 \%$ of the variance in employees' job dissatisfaction is explained by the independent variables. Once taking into account the control variables, the effect of digital work intensification on employees' job dissatisfaction decreases slightly (from 0.188 to 0.180 ).

Regarding our first research question, the results show that higher levels of digital work intensification significantly relate to higher job dissatisfaction even when accounting for occupation-specific ICT use, education, and other important spurious variables (Hypothesis 1a). Furthermore, we found evidence that the more frequently employees use enterprise application systems for production (e.g., Product Lifecycle Management Systems, Manufacturing Execution Systems, and Production Planning and Control Systems), the more dissatisfied they are in their jobs (Hypothesis 1b). However, all other considered forms of occupation-specific ICT show no significant impact.

The aim of Model 3 is to investigate how far the effect of digital work intensification decreases when employees' digital self-efficacy is included in the model. Including employees' digital self-efficacy also

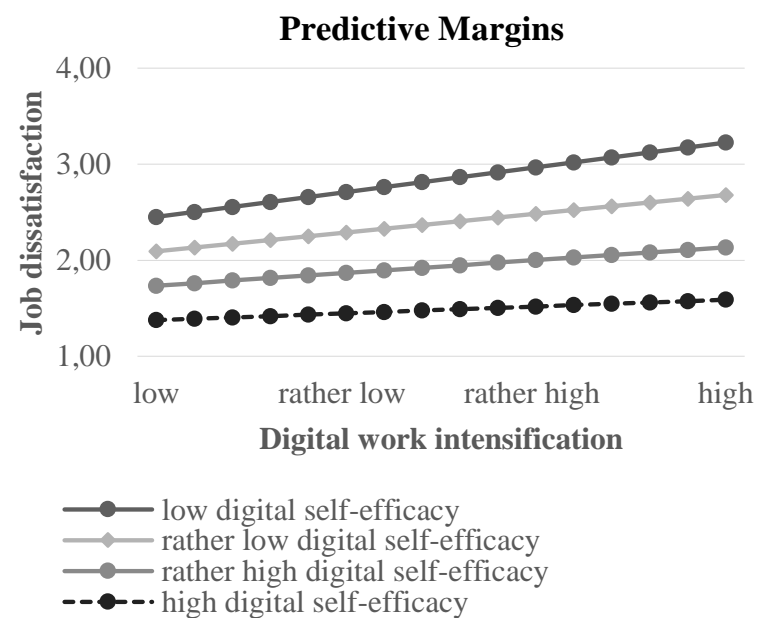

significantly improved the model fit for the regression model (likelihood ratio test $[p<0.001]$ ), accounting for $23.4 \%$ of the variance of employees' perceived job dissatisfaction. With regard to our second research question (RQ2), the effect of digital work intensification decreases by approximately 55\% when employees' digital self-efficacy is included in the model. In addition, digital self-efficacy is shown to be the strongest predictor in the model of employees' job dissatisfaction. Furthermore, our results indicate that employees who use communication applications (e.g., e-mail, messenger, videoconferencing tools) more frequently significantly perceived less job dissatisfaction, net of all controls.

To determine whether the effect of digital work intensification on job dissatisfaction varies across different levels of digital self-efficacy (RQ2, Hypothesis 2), we added an interaction between the measures for digital work intensification and digital self-efficacy to the regression model. As interactions are often difficult to interpret from regression coefficients, the results are displayed in Figure 3 to clarify the interpretation. Figure 3 depicts the effect of digital work intensification on job dissatisfaction for different levels of digital self-efficacy. Note that the estimates of Figure 3 are adjusted for all covariates of Model 3.

As seen in the right graph in Figure 3, the effect size of digital work intensification decreases from $0.26^{* *}$ to $0.07 *$ as employee's digital self-efficacy increases. In other words, the extent to which digital work intensification is associated with higher job dissatisfaction is substantially lower for employees with higher digital self-efficacy than for employees with lower digital self-efficacy.

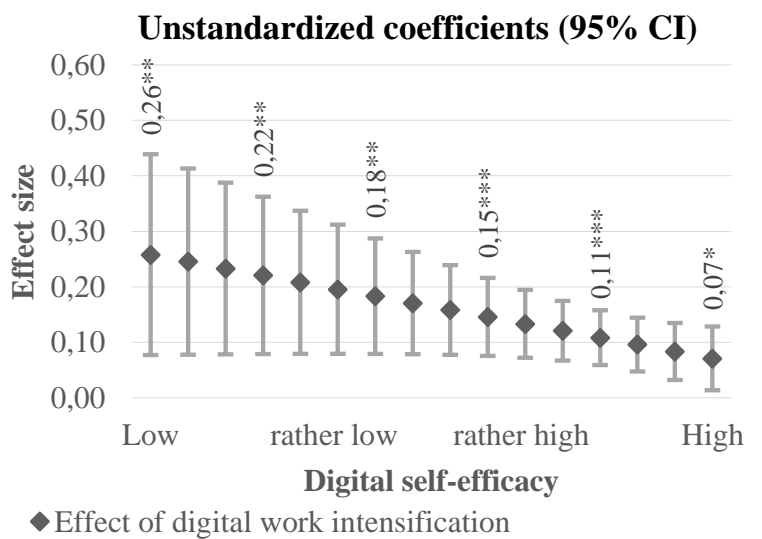

$\diamond$ Effect of digital work intensification

\section{Figure 3. Effect of digital work intensification on job dissatisfaction at different levels of digital self-efficacy}


For easier interpretation, the left graph in Figure 3 displays the predicted values of employees' job dissatisfaction according to their level of digital work intensification and digital self-efficacy. Overall, job dissatisfaction rises as the level of digital work intensification increases. However, this is especially the case for employees with (rather) low digital selfefficacy. In contrast, the line for employees with the highest level of digital self-efficacy is nearly flat (left graph in Figure 3), indicating that higher levels of digital work intensification have almost no impact on their job dissatisfaction. In addition, employees with lower digital self-efficacy are more dissatisfied with their job when digital work intensification due to ICT exposure is high than employees with higher digital self-efficacy. In sum, the results suggest that digital selfefficacy buffers the effect of digital work intensification on job dissatisfaction.

\section{Discussion}

The aim of this article was twofold. On the one hand, we investigated to what extent ICT exposure impacts employees' job dissatisfaction. Our results provide evidence that employees who perceive higher work intensification through ICT also perceive higher job dissatisfaction, thereby confirming Hypothesis 1a.

However, we found no evidence that individuals who use ICT to a higher degree than others also perceive higher job dissatisfaction. Thus, Hypothesis $1 \mathrm{~b}$ is not confirmed. The use of communication applications even leads to a reverse effect and is associated with lower job dissatisfaction. On the other hand, we examined if employees' digital self-efficacy buffers the association between digital work intensification and employees' job dissatisfaction. Our findings provide evidence for the buffering hypothesis. Especially for employees with low digital self-efficacy, higher degrees of digital work intensification were associated with higher degrees of job dissatisfaction. These findings suggest that digital self-efficacy may reduce the negative consequences of digital work intensification on employees' job well-being. Thereby our results lend support for Hypothesis 2.

Our results contribute to the literature in several ways. To the best of our knowledge, this is the first study related to the impact of ICT exposure on job dissatisfaction considering the buffering effect of digital self-efficacy. Ensuring high data quality by using an ISO-certified online panel, as well as validated scales, provide the study with a promising and representative data basis for drawing well-founded conclusions of the surveyed group of employees.

The distinction between digital work intensification and various forms of occupation-specific
ICT helped us to separate the effects of the mere use of ICT and the perceived stress associated with its use. We found evidence that higher levels of digital work intensification are related to higher levels of employees' job dissatisfaction, regardless of the intensity, employees work with ICT. These insights contribute to the body of research on the phenomena of work intensification by shedding light on the influences of ICT that contribute to poor employee outcomes. Further, we revealed that the mere use of occupation-specific ICT does not affect employees' job dissatisfaction in a negative manner. The increased use of communication applications even has the opposite effect, which is divergent from the major body of previous findings in research, in which the use of email is associated with extensive workloads and job strain [e.g., 1, 4, 47]. A possible explanation for this effect may be the social component of communication applications as they foster social interactions with colleagues that play an essential role in employees' well-being and have been shown to improve job satisfaction [48]. In addition, positive interactions between supportive co-workers can reduce role ambiguity and workload that increase job satisfaction as well as organizational commitment [49].

This reasoning is consistent with the results of this study, which highlight the importance of factors that buffer the negative impact of ICT exposure. We know from literature that situational factors can weaken the effect of ICT exposure and reduce them such as job control and social support $[2,8]$. However, very limited research exists so far on a possible moderating effect of inhibitors of ICT exposure [21]. Therefore, we contribute to the body of research by underlining that digital self-efficacy is a strong personal disposition that moderates the negative effect of intensified workloads caused by ICT on employees' well-being as job dissatisfaction. Our results show that the effect of perceived digital work intensification reduces with increasing levels of digital self-efficacy. In other words, the lower the employees' digital self-efficacy, the more dissatisfied they are in their jobs as digital work intensification increases. In this respect, our results highlight that employees' beliefs or motivation are important factors in coping with the demands of ICT exposure and foster employees' well-being. We believe that this finding is theoretically important and introduces richness in the emerging literature on the dark side of ICT use.

Thus, our study does not only contribute to theory but also allows us to draw implications for practice. Based on the outstanding importance of the concept of digital self-efficacy in our study, organizations should consider measures aimed at promoting employees to understand and learn to cope with the challenges and requirements of a digital working world. The literature 
shows that training programs are important means to improve forms of digital self-efficacy [8, 20, 37]. Furthermore, organizations should take important complementary factors into account that lead to better cope with the ICT-related work stress as well. These include organizational aspects like help desk support, but also corporate cultural aspects like a positive and supportive team climate as well as attentive leadership [8].

In terms of limitations of our study, first, subjective, self-report measures were used. Second, our sample is limited in geographical scope to a national representative cross-section of service and commercial occupations of the German labor market. Third, we conducted data collection before the COVID-19 pandemic led to increased remote work in German organizations. Therefore, this effect does not affect our results, though they do not bias them either. Nevertheless, our results may have become even more virulent during the pandemic. The increased remote working may not only increase digital work intensification. Also, social network support in organizations may have suffered due to reduced social interactions between employees. Both can potentially amplify the negative effects of ICT exposure. Fourth, as we consider digital self-efficacy as the only buffering effect in our study, future studies can integrate several inhibiting factors to shed light on the associations between different inhibitors (e.g., social support). Fifth, since the obtained findings are based on cross-sectional data, we cannot draw causal conclusions. Therefore, future research should focus on intervention studies to measure the consequences of ICT exposure over time and in tandem with inhibiting factors like digital self-efficacy $[3,5,8]$.

\section{Conclusion}

The goal of this study was to analyze the association between ICT exposure and job dissatisfaction considering a possible buffering effect of employees' digital self-efficacy. To achieve this goal, we conducted a quantitative study with a national representative sample of commercial and service occupations. Our results revealed that employees who perceive higher levels of digital work intensification are more dissatisfied in their jobs. We also demonstrated that employees with higher digital self-efficacy can moderate the positive effects of work intensification on job dissatisfaction, underscoring the importance of the psychological concept of self-efficacy in both theoretical and practical discourse. Returning to the title of the paper, our results suggest that technology doesn't matter too much if employees have a positive self-perception of their abilities to cope with the requirements of digital work.

\section{References}

[1] Ayyagari, R., V. Grover, and R. Purvis, "Technostress: Technological Antecedents and Implications", MIS Quarterly, 35(4), 2011, pp. 831-858.

[2] Ragu-Nathan, T.S., M. Tarafdar, B.S. Ragu-Nathan, and Q. Tu, "The Consequences of Technostress for End Users in Organizations: Conceptual Development and Empirical Validation", Information Systems Research, 19(4), 2008, pp. 417-433.

[3] Berg-Beckhoff, G., G. Nielsen, and E. Ladekjær Larsen, "Use of information communication technology and stress, burnout, and mental health in older, middle-aged, and younger workers - results from a systematic review", International Journal of Occupational and Environmental Health, 23(2), 2017, pp. 160-171.

[4] Atanasoff, L. and M.A. Venable, "Technostress: Implications for Adults in the Workforce", The Career Development Quarterly, 65(4), 2017, pp. 326-338.

[5] Benzari, A., A. Khedhaouria, and O. Torrès, "The Rise of Technostress: A Literature Review from 1984 until 2018", in ECIS 2020 Research Papers, A Virtual AIS Conference. 2020. [6] Tarafdar, M., Q. Tu, B.S. Ragu-Nathan, and T.S. RaguNathan, "The Impact of Technostress on Role Stress and Productivity", Journal of Management Information Systems, 24(1), 2007, pp. 301-328.

[7] Tarafdar, M., Q. Tu, T.S. Ragu-Nathan, and B.S. RaguNathan, "Crossing to the dark side", Communications of the ACM, 54(9), 2011, pp. 113-120.

[8] Tarafdar, M., E.B. Pullins, and T.S. Ragu-Nathan, "Technostress: negative effect on performance and possible mitigations", Information Systems Journal, 25(2), 2015, pp. 103-132.

[9] Tarafdar, M., Q. Tu, and T.S. Ragu-Nathan, "Impact of Technostress on End-User Satisfaction and Performance", Journal of Management Information Systems, 27(3), 2010, pp. 303-334.

[10] Yin, P., C.X.J. Ou, R.M. Davison, and J. Wu, "Coping with mobile technology overload in the workplace", Internet Research, 28(5), 2018, pp. 1189-1212.

[11] Borle, P., F. Boerner-Zobel, S. Voelter-Mahlknecht, H.M. Hasselhorn, and M. Ebener, "The social and health implications of digital work intensification. Associations between exposure to information and communication technologies, health and work ability in different socioeconomic strata", International Archives of Occupational and Environmental Health, 94(3), 2021, pp. 377-390.

[12] Brod, C., Technostress: The human cost of the computer revolution, Addison-Wesley, Reading, Mass., 1984.

[13] Day, A., S. Paquet, N. Scott, and L. Hambley, "Perceived information and communication technology (ICT) demands on employee outcomes: The moderating effect of organizational ICT support", Journal of Occupational Health Psychology, 17(4), 2012, pp. 473-491.

[14] Shu, Q., Q. Tu, and K. Wang, "The Impact of Computer Self-Efficacy and Technology Dependence on ComputerRelated Technostress: A Social Cognitive Theory 
Perspective", International Journal of Human-Computer Interaction, 27(10), 2011, pp. 923-939.

[15] Lazarus, R.S., Psychological stress and the coping process, McGraw-Hill, New York, 1966.

[16] Chesley, N., "Information and communication technology use, work intensification and employee strain and distress", Work, Employment and Society, 28(4), 2014, pp. 589-610.

[17] Borle, P., K. Reichel, and S. Voelter-Mahlknecht, "Is There a Sampling Bias in Research on Work-Related Technostress? A Systematic Review of Occupational Exposure to Technostress and the Role of Socioeconomic Position", International journal of environmental research and public health, 18(4), 2021, pp. 1-22.

[18] Maier, C., S. Laumer, and C. Weinert, "Enterprise Resource Planning Systems Induced Stress: a Comparative Empirical Analysis with Young and Elderly SAP Users", in Wirtschaftsinformatik Proceedings 2015, Osnabrück. 2015.

[19] Conway, F.T., "Psychological Mood State, Psychosocial Aspects of Work, and Musculoskeletal Discomfort in Intensive Video Display Terminal (VDT) Work", International Journal of Human-Computer Interaction, 11(2), 1999, pp. 95-107.

[20] Torkzadeh, G. and T.P. van Dyke, "Effects of training on Internet self-efficacy and computer user attitudes", Computers in Human Behavior, 18(5), 2002, pp. 479-494.

[21] Sarabadani, J., M. Carter, and D. Compeau, "10 Years of Research on Technostress Creators and Inhibitors: Synthesis and Critique", in Twenty-fourth Americas Conference on Information Systems, New Orleans. 2018.

[22] Judge, T.A. and R. Klinger, "Job Satisfaction: Subjective Well-Being at Work", in The science of subjective well-being, M. Eid and R.J. Larsen, Editors. 2008. Guilford Press: New York.

[23] Locke, E., "The nature and causes of job satisfaction", in Handbook of Industrial and Organizational Psychology. 1976. Wiley: New York.

[24] Green, F., "Why Has Work Effort Become More Intense?", Industrial Relations, 43(4), 2004, pp. 709-741.

[25] Kelliher, C. and D. Anderson, "For better or for worse? An analysis of how flexible working practices influence employees' perceptions of job quality", The International Journal of Human Resource Management, 19(3), 2008, pp. 419-431.

[26] Green, F. and S. McIntosh, "The intensification of work in Europe", Labour Economics, 8(2), 2001, pp. 291-308.

[27] Handel, M.J., "Trends in Perceived Job Quality, 1989 to 1998", Work and Occupations, 32(1), 2005, pp. 66-94.

[28] https://s.gwdg.de/QyBF6y, accessed 2-12-2021.

[29] Morris, M.G. and V. Venkatesh, "Job Characteristics and Job Satisfaction: Understanding the Role of Enterprise Resource Planning System Implementation", MIS Quarterly, 34(1), 2010, pp. 143-161.

[30] Zečević, A., J. Radović-Stojanović, and A. Čudan, "The use of information and communication technologies by enterprises in the European Union member countries", Ekonomski horizonti, 21(3), 2019, pp. 273-294.

[31] Waizenegger, L., B. McKenna, W. Cai, and T. Bendz, "An affordance perspective of team collaboration and enforced working from home during COVID-19", European Journal of Information Systems, 29(4), 2020, pp. 429-442.
[32] Brown, M., "Responses to work intensification: does generation matter?", The International Journal of Human Resource Management, 23(17), 2012, pp. 3578-3595.

[33] Bandura, A., "Self-efficacy: Toward a unifying theory of behavioral change", Psychological Review, 84(2), 1977, pp. 191-215.

[34] Bandura, A., Social foundations of thought and action: A social cognitive theory, Prentice-Hall, Englewood Cliffs, 1986.

[35] Moos, D.C. and R. Azevedo, "Learning With ComputerBased Learning Environments: A Literature Review of Computer Self-Efficacy", Review of Educational Research, 79(2), 2009, pp. 576-600.

[36] Lee, Y.-K., "Impacts of Digital Technostress and Digital Technology Self-Efficacy on Fintech Usage Intention of Chinese Gen Z Consumers", Sustainability, 13(9), 2021, pp. 1-15.

[37] Compeau, D.R. and C.A. Higgins, "Computer SelfEfficacy: Development of a Measure and Initial Test", MIS Quarterly, 19(2), 1995, pp. 189-211.

[38] Härtwig, C., K. Borgnäs, S. Tuleweit, A. Lenski, and Niebuhr. C., Beschäftigtenbefragung Monitor Digitalisierung: Entwicklungen der Arbeitsqualität in zwölf Industriebranchen, Stiftung Arbeit und Umwelt der IG BCE, Berlin, 2019.

[39] Leiner, D.J., "Too Fast, too Straight, too Weird: NonReactive Indicators for Meaningless Data in Internet Surveys", Survey Research Methods, 13(3), 2019, pp. 229-248.

[40] https://s.gwdg.de/jykguK, accessed 2-2-2021.

[41] Cronbach, L.J., "Coefficient alpha and the internal structure of tests", Psychometrika, 16(3), 1951, pp. 297-334.

[42] Enzmann, D. and D. Kleiber, Helfer-Leiden: Streß und Burnout in psychosozialen Berufen, Asanger, Heidelberg, 189.

[43] Muthén, B.O., L.K. Muthén, and T. Asparouhov, Regression and mediation analysis using Mplus, Muthén \& Muthén, Los Angeles, CA, 2016.

[44] Stegmann, S., R. van Dick, J. Ullrich, J. Charalambous, B. Menzel, N. Egold, and T.T.-C. Wu, "Der Work Design Questionnaire", Zeitschrift für Arbeits- und Organisationspsychologie A\&O, 54(1), 2010, pp. 1-28.

[45] Jerusalem, M. and R. Schwarzer, "Selbstwirksamkeit [Self-efficacy]", in Skalen zur Befindlichkeit und Persönlichkeit, R. Schwarzer, Editor. 1986. Freie Universität, Institut für Psychologie: Berlin.

[46] Neyer, F.J., J. Felber, and C. Gebhardt, "Entwicklung und Validierung einer Kurzskala zur Erfassung von Technikbereitschaft (technology commitment)", Diagnostica, 58(2), 2012, pp. 87-99.

[47] Stich, J.-F., M. Tarafdar, P. Stacey, and C.L. Cooper, "Email load, workload stress and desired e-mail load: a cybernetic approach", Information Technology \& People, 32(2), 2019, pp. 430-452.

[48] Hodson, R., "Work Life and Social Fulfillment: Does Social Affiliation at Work Reflect a Carrot or a Stick?", Social Science Quarterly, 85(2), 2004, pp. 221-239.

[49] Chiaburu, D.S. and D.A. Harrison, "Do peers make the place? Conceptual synthesis and meta-analysis of coworker effects on perceptions, attitudes, OCBs, and performance", Journal of Applied Psychology, 93(5), 2008, pp. 1082-1103. 